\title{
AN EASY, EFFICIENT PTC-MEDIATED SYNTHESIS OF 2- SUBSTITUTED-6-CHLOROQUINOXALINES AND ANTIBACTERIAL ACTIVITY
}

\author{
T. Siva Sankara Babu ${ }^{1}$, N. Srinivasu ${ }^{1, *}$, B. Saha ${ }^{2}$ and S. Venkat Reddy ${ }^{2}$ \\ ${ }^{1}$ Division of Chemistry, Department of Science and Humanities, Vignan's Foundation for \\ Science, Technology and Research University, Guntur, India. \\ ${ }^{2}$ NACL Industries Limited(R\&D Center), Hyderabad, India. \\ *E-mail: navulurisrinivasu@gmail.com
}

\begin{abstract}
An easy and efficient synthetic protocol has been developed for the synthesis of 2-substituted-6-Chloro-quinoxalines by the reaction of 2,6-Dichloroquinoxaline with different alcohols, thiols and amine using Tri Ethyl Benzyl Ammonium Chloride (TEBAC). The newly synthesized compounds characterized by spectral data such as IR, ${ }^{1} \mathrm{HNMR},{ }^{13} \mathrm{CNMR}$ and mass spectrometry. The compounds were screened for their in vitro antibacterial activity against four different organisms, resulted in the compounds showed good antibacterial activities.
\end{abstract}

Keywords: 6-Chloroquinoxaline, Phase Transfer Catalysts, Thiols, Amine, Antibacterial Activity.

(C) RASĀYAN. All rights reserved

\section{INTRODUCTION}

Quinoxalines are an important class of heterocyclic compounds due to their importance in the fields such as medical, industrial and synthetic organic chemistry. ${ }^{1}$ Quinoxaline derivatives are exhibit a variety of biological activities including antifungal, ${ }^{2}$ antioxidant, ${ }^{2}$ antibacterial, ${ }^{3}$ anticancer ${ }^{4}$ and antidiabetic. ${ }^{5}$ Quinoxaline derivatives are also applicable in the macrocyclic receptor, electroluminescent, organic semiconductor and DNA cleavage study. ${ }^{6}$ Echinomycin, Neomycin and Actinoleucin are marketed antibiotic drugs which structure consists of quinoxaline core moiety ${ }^{7}$ and many synthetic quinoxaline derivatives are used to treat tuberculosis. ${ }^{8}$ The activities of the quinoxaline compound are enhanced by introducing a different substitution pattern. Also, many heterocyclic compounds with heteroatom substitution on their ring system sulfur usually are a good antifungal agent. Halogens are played an important role in drug designing over the last 30 years. In modern drug discovery, the inclusion of a chlorine atom into a drug molecule is most significant due to that distribution in a fatty and aqueous medium. Given the above facts, we have planned to synthesis 2-substituted-6-Chloro-quinoxalines (Scheme-1).

\section{Material and Methods}

\section{EXPERIMENTAL}

All Reagent grade chemicals are used in this work. Chemicals were purchased from Sigma-Aldrich Chemicals Co. and Spectrochem Pvt. Ltd. These reagent grade chemicals were used without further purification. Solvents were dried according to standard methods. Melting points were determined in open capillary tubes and are uncorrected. IR spectrum was taken on a JASCO FT-IR-4100 using KBr pellet method. ${ }^{1} \mathrm{H} \&{ }^{13} \mathrm{C}-\mathrm{NMR}$ spectra were recorded on a JEOL $400 \mathrm{MHz}$ and BRUKER $500 \mathrm{MHz}$ spectrometers in $\mathrm{CDCl}_{3}$ using TMS as an internal reference, $J$ in Hertz. MS was performed using SHIMADZU LC-2010EV spectrometer.

\section{General Procedure for the Synthesis of 2-Substituted 6-Chloroquinoxaline Derivatives (3a-f)}

To a stirred solution of 2,6-Dichloroquinoxaline (1) $(0.085 \mathrm{~mol}), \mathrm{R}-\mathrm{X}(\mathbf{2 a - e})(0.085 \mathrm{~mol})$ in N, NDimethyl formamide $(25 \mathrm{~mL})$ was added TEBAC $(0.0085 \mathrm{~mol})$ and $\mathrm{K}_{2} \mathrm{CO}_{3}(0.093 \mathrm{~mol})$ at room

Rasayan J. Chem., 13(2), 1037-1041(2020)

http://dx.doi.org/10.31788/RJC.2020.1325693

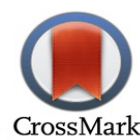


RASĀYAN J. Chem.

Vol. 13 | No. 2 |1037 - 1041| April - June | 2020

temperature and heated to $70-75^{\circ} \mathrm{C}$ and stirred for $6-7 \mathrm{hrs}$. After completion of the reaction (monitored by TLC), the reaction mixture filtered, the filtrate was added to ice-cold water and the compound extracted with ethyl acetate $(2 \times 25 \mathrm{ml})$. The organic layer dried over $\mathrm{Na}_{2} \mathrm{SO}_{4}$ and concentrated under reduced pressure to obtained crude product, then the product purified by column chromatography using silica gel using $25 \%$ Ethyl acetate in n-hexane to afford pure 2-Substituted 6-Chloroquinoxaline derivatives (3a-e).

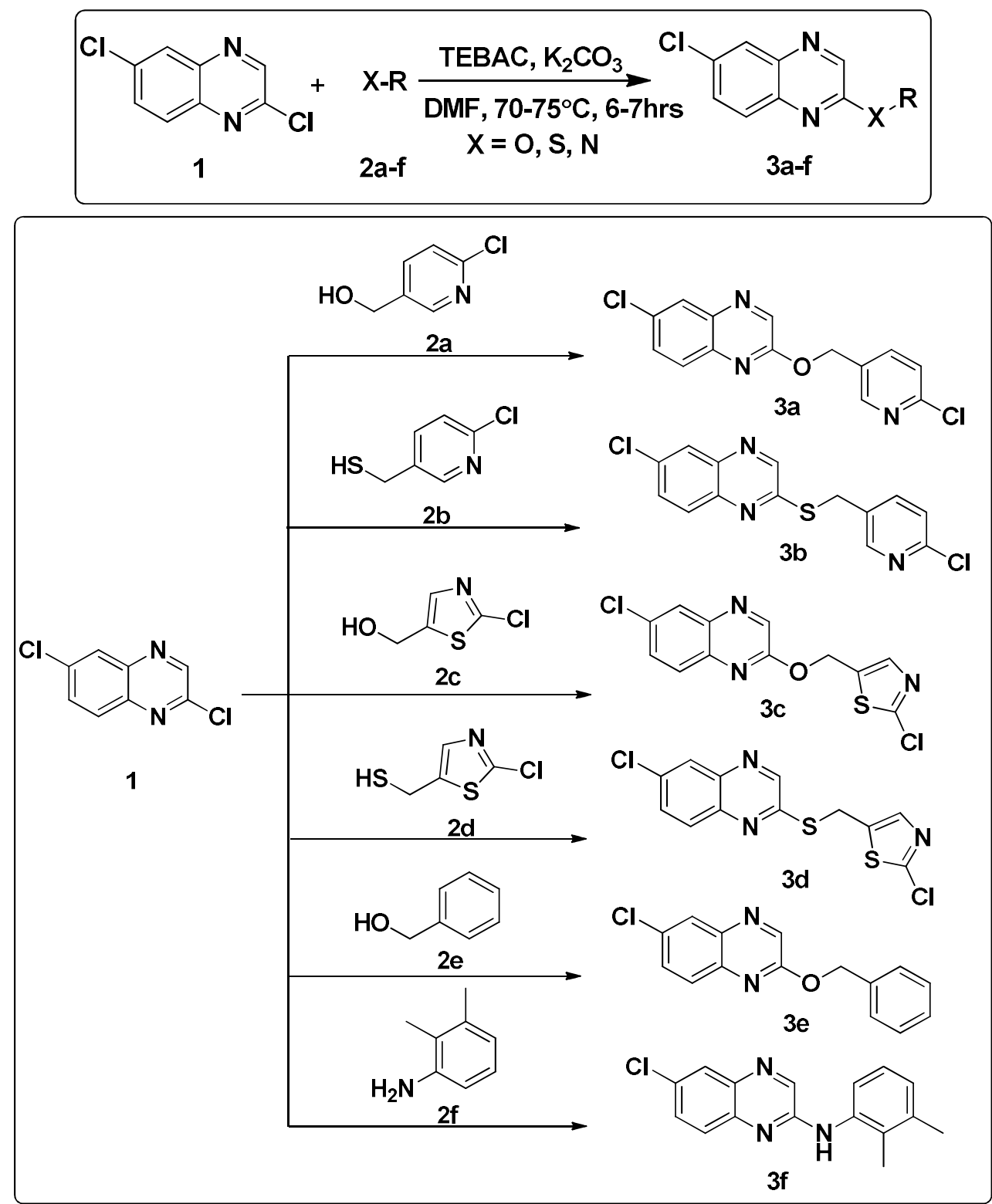

Scheme-1: Synthesis of 2-Substituted 6-chloroquinoxaline derivatives (3a-f)

\section{6-Chloro-2-((6-chloropyridin-3-yl)methoxy)quinoxaline (3a)}

Pale Yellow solid, Yield: 84\%; mp 149-151 ${ }^{\circ} \mathrm{C}$; IR spectrum, $v, \mathrm{~cm}^{-1}$ : 3043, 2924, 1590, 1488, 1384, 828; ${ }^{1} \mathrm{H}$ NMR spectrum, $\delta$, ppm $(J, \mathrm{~Hz}): 5.53 \mathrm{~s}(2 \mathrm{H}), 7.37 \mathrm{~d}\left(J=8.3 \mathrm{~Hz}, 1 \mathrm{H}, \mathrm{C}_{6} \mathrm{H}_{5}\right), 7.63-7.66 \mathrm{dd}(J=$ $\left.9.0,2.8 \mathrm{~Hz}, 1 \mathrm{H}, \mathrm{C}_{6} \mathrm{H}_{5}\right), 7.78 \mathrm{~d}\left(J=9.0 \mathrm{~Hz}, 1 \mathrm{H}, \mathrm{C}_{6} \mathrm{H}_{5}\right), 7.82-7.84 \mathrm{dd}\left(J=8.3,2.8, \mathrm{~Hz}, 1 \mathrm{H}, \mathrm{C}_{6} \mathrm{H}_{5}\right), 8.03 \mathrm{~d}(J$ $\left.=2.1 \mathrm{~Hz}, 1 \mathrm{H}, \mathrm{C}_{6} \mathrm{H}_{5}\right), 8.52 \mathrm{~s}\left(1 \mathrm{H}, \mathrm{C}_{6} \mathrm{H}_{5}\right), 8.58 \mathrm{~d}\left(J=2.1 \mathrm{~Hz}, 1 \mathrm{H}, \mathrm{C}_{6} \mathrm{H}_{5}\right) ;{ }^{13} \mathrm{C}$ NMR spectrum, $\delta$, ppm: 156.6, 151.6, 149.9, 140.2, 139.5, 139.0, 138.6, 132.6, 131.2, 130.8, 128.4, 128.2, 124.3, 64.9; ESI-MS: Found $306.03[M+\mathrm{H}]+$ and calculated for $\mathrm{C}_{14} \mathrm{H}_{9} \mathrm{Cl}_{2} \mathrm{~N}_{3} \mathrm{O}: 306.15$ 


\section{6-Chloro-2-(((6-chloropyridin-3-yl)methyl)thio)quinoxaline (3b)}

Pale Yellow solid, Yield: 88\%; mp 127-129 ${ }^{\circ} \mathrm{C}$; IR spectrum, $v, \mathrm{~cm}^{-1}$ : 3048, 2931, 1604, 1585,1536, $1461,828,781 ;{ }^{1} \mathrm{H}$ NMR spectrum, $\delta$, ppm $(J, \mathrm{~Hz}): 4.50 \mathrm{~s}(2 \mathrm{H}), 7.27 \mathrm{~m}\left(1 \mathrm{H}, \mathrm{C}_{6} \mathrm{H}_{5}\right), 7.65-7.68 \mathrm{dd}(J=$ $\left.2.3,8.9 \mathrm{~Hz}, 1 \mathrm{H}, \mathrm{C}_{6} \mathrm{H}_{5}\right), 7.76-7.78 \mathrm{dd}\left(J=2.5,8.2 \mathrm{~Hz}, 1 \mathrm{H}, \mathrm{C}_{6} \mathrm{H}_{5}\right), 7.89 \mathrm{~d}\left(J=8.9 \mathrm{~Hz}, 1 \mathrm{H}, \mathrm{C}_{6} \mathrm{H}_{5}\right), 8.01 \mathrm{~d}(J$ $\left.=2.3 \mathrm{~Hz}, 1 \mathrm{H}, \mathrm{C}_{6} \mathrm{H}_{5}\right), 8.56 \mathrm{~d}\left(\mathrm{~J}=2.5 \mathrm{~Hz}, 1 \mathrm{H}, \mathrm{C}_{6} \mathrm{H}_{5}\right), 8.58 \mathrm{~s}\left(1 \mathrm{H}, \mathrm{C}_{6} \mathrm{H}_{5}\right) ;{ }^{13} \mathrm{C}$ NMR spectrum, $\delta$, ppm: 154.7 , $150.5,150.3,145.2,141.0,140.5,139.4,134.1,132.5,131.4,128.9,128.4,124.2,30.1$; ESI-MS: Found $322.20[M+\mathrm{H}]+$ and Calculated for $\mathrm{C}_{14} \mathrm{H}_{9} \mathrm{Cl}_{2} \mathrm{~N}_{3} \mathrm{~S}: 322.21$

\section{2-Chloro-5-(((6-chloroquinoxalin-2-yl)oxy)methyl)thiazole (3c)}

Off-White solid, Yield: $85 \%$; mp 107-109 ${ }^{\circ}$; IR spectrum, $v, \mathrm{~cm}^{-1}: 3086,3047,2958,1606,1573,1529$, 1305, 1054, 824; ${ }^{1} \mathrm{H}$ NMR spectrum, $\delta$, ppm: $(J, \mathrm{~Hz}): 5.63 \mathrm{~s}(2 \mathrm{H}), 7.69 \mathrm{~m}\left(2 \mathrm{H}, \mathrm{C}_{6} \mathrm{H}_{5}\right), 7.84 \mathrm{~d}(J=8.8 \mathrm{~Hz}$, $\left.1 \mathrm{H}, \mathrm{C}_{6} \mathrm{H}_{5}\right), 8.05 \mathrm{~d}\left(J=2.3 \mathrm{~Hz}, 1 \mathrm{H}, \mathrm{C}_{6} \mathrm{H}_{5}\right), 8.50 \mathrm{~s}\left(1 \mathrm{H}, \mathrm{C}_{6} \mathrm{H}_{5}\right),{ }^{13} \mathrm{C}$ NMR spectrum, $\delta$, ppm: $155.9,153.8$, 142.1, 140.0, 139.3, 138.0, 134.4, 132.5, 131.2, 128.1, 128.0, 59.9; ESI-MS: Found $311.98[M+\mathrm{H}]+$ and Calculated for $\mathrm{C}_{12} \mathrm{H}_{7} \mathrm{C}_{12} \mathrm{~N}_{3} \mathrm{OS}: 312.17$.

\section{2-Chloro-5-(((6-chloroquinoxalin-2-yl)thio)methyl)thiazole (3d)}

Off-White solid, Yield: $90 \%$; mp $160-161^{\circ} \mathrm{C}$; IR spectrum, $v, \mathrm{~cm}^{-1}$ : 3054, 2985, 1603, 1536, 1477, 1087, 823; ${ }^{1} \mathrm{H}$ NMR spectrum, $\delta$, ppm: $(J, \mathrm{~Hz}): 4.64 \mathrm{~s}(2 \mathrm{H}), 7.53 \mathrm{~s}\left(1 \mathrm{H}, \mathrm{C}_{6} \mathrm{H}_{5}\right), 7.70-7.72 \mathrm{dd}(J=2.4$, $\left.8.6 \mathrm{~Hz}, 1 \mathrm{H}, \mathrm{C}_{6} \mathrm{H}_{5}\right), 7.95 \mathrm{~d}\left(J=9.0 \mathrm{~Hz}, 1 \mathrm{H}, \mathrm{C}_{6} \mathrm{H}_{5}\right), 8.05 \mathrm{~d}\left(J=2.8 \mathrm{~Hz}, 1 \mathrm{H}, \mathrm{C}_{6} \mathrm{H}_{5}\right), 8.60 \mathrm{~s}\left(1 \mathrm{H}, \mathrm{C}_{6} \mathrm{H}_{5}\right) ;{ }^{13} \mathrm{C}$ NMR spectrum, $\delta$, ppm: $154.4,152.1,145.2,140.8,140.5,140.4,137.3,134.3,131.6,128.9,128.5,25.8$; ESI-MS: Found $327.95[M+\mathrm{H}]+$. Calculated for $\mathrm{C}_{12} \mathrm{H}_{7} \mathrm{Cl}_{2} \mathrm{~N}_{3} \mathrm{~S}_{2}: 328.24$.

\section{2-(Benzyloxy)-6-chloroquinoxaline (3e)}

Pale Yellow solid, Yield: $83 \%$; $\mathrm{mp} 97-100^{\circ} \mathrm{C}$; IR spectrum, $v, \mathrm{~cm}^{-1}: 3043,2924,2853,1603,1565,1009$, 878; ${ }^{1} \mathrm{H}$ NMR spectrum, $\delta$, ppm: $(J, \mathrm{~Hz}): 5.52 \mathrm{~s}(2 \mathrm{H}), 7.35-7.43 \mathrm{~m}\left(3 \mathrm{H}, \mathrm{C}_{6} \mathrm{H}_{5}\right), 7.52 \mathrm{~d}(J=7.6 \mathrm{~Hz}, 2 \mathrm{H}$, $\left.\mathrm{C}_{6} \mathrm{H}_{5}\right), 7.62-7.64 \mathrm{dd}\left(J=2.4,8.6 \mathrm{~Hz}, 1 \mathrm{H}, \mathrm{C}_{6} \mathrm{H}_{5}\right), 7.80 \mathrm{~d}\left(J=9.0 \mathrm{~Hz}, 1 \mathrm{H}, \mathrm{C}_{6} \mathrm{H}_{5}\right), 8.02 \mathrm{~d}(J=2.8 \mathrm{~Hz}, 1 \mathrm{H}$, $\left.\mathrm{C}_{6} \mathrm{H}_{5}\right), 8.52 \mathrm{~s}\left(1 \mathrm{H}, \mathrm{C}_{6} \mathrm{H}_{5}\right) ;{ }^{13} \mathrm{C}$ NMR spectrum, $\delta$, ppm: $157.3,140.7,139.3,138.9,136.1,132.1,131.0$, 128.7, 128.6, 128.5, 128.2, 68.5; ESI-MS: Found $271.03[M+\mathrm{H}]+$ and Calculated for $\mathrm{C}_{15} \mathrm{H}_{11} \mathrm{ClN}_{2} \mathrm{O}$ : 270.71 .

\section{Procedure for the synthesis of compound (3f)}

A mixture of 2, 6-Dichloro Quinoxaline (1) $(0.125 \mathrm{~mol})$ and 2,3-Dimethylaniline (2f) $(0.125 \mathrm{~mol})$ were taken in N,N-Dimethylformamide $(25 \mathrm{~mL})$ at room temperature. Then the reaction mixture was heated to $100^{\circ} \mathrm{C}$ and was maintained for $4.5 \mathrm{hrs}$. Then the crude product purified by column chromatography using silica gel using 5\% Ethyl acetate in n-hexane to afforded 6-chloro-N-(2,3-dimethylphenyl)quinoxalin-2amine (3f) as off white solid.

\section{6-chloro-N-(2,3-dimethylphenyl)quinoxalin-2-amine (3f)}

Reddish brown solid, Yield: $86 \%$; mp $138-140^{\circ} \mathrm{C}$; IR spectrum, $v, \mathrm{~cm}^{-1}: 3199,3054,2920,1618,1585$, 1471, 1260, 976, 821; ${ }^{1} \mathrm{H}$ NMR spectrum, $\delta$, ppm: $(J, \mathrm{~Hz}): 2.24 \mathrm{~s}\left(3 \mathrm{H}, \mathrm{CH}_{3}\right), 2.36 \mathrm{~s}\left(3 \mathrm{H}, \mathrm{CH}_{3}\right), 6.67$ br s $(1 \mathrm{H}), 7.11 \mathrm{~d}\left(J=7.6 \mathrm{~Hz}, 1 \mathrm{H}, \mathrm{C}_{6} \mathrm{H}_{5}\right), 7.16-7.19 \mathrm{t}\left(J=7.7 \mathrm{~Hz}, 1 \mathrm{H}, \mathrm{C}_{6} \mathrm{H}_{5}\right), 7.44 \mathrm{~d}\left(J=7.7 \mathrm{~Hz}, 1 \mathrm{H}, \mathrm{C}_{6} \mathrm{H}_{5}\right)$, $7.53-7.55 \mathrm{dd}\left(J=9.0,2.4 \mathrm{~Hz}, 1 \mathrm{H}, \mathrm{C}_{6} \mathrm{H}_{5}\right), 7.63 \mathrm{~d}\left(J=9.0 \mathrm{~Hz}, 1 \mathrm{H}, \mathrm{C}_{6} \mathrm{H}_{5}\right), 7.90 \mathrm{~d}\left(J=2.2 \mathrm{~Hz}, 1 \mathrm{H}, \mathrm{C}_{6} \mathrm{H}_{5}\right)$, $8.34 \mathrm{~s}\left(1 \mathrm{H}, \mathrm{C}_{6} \mathrm{H}_{5}\right) ;{ }^{13} \mathrm{C}$ NMR spectrum, $\delta$, ppm: $151.0,140.2,138.5,138.1,136.3,131.5,130.9,130.3$, 128.0, 127.9, 127.5, 126.4, 122.6, 20.7, 14.2; ESI-MS: Found 284.05 $[M+\mathrm{H}]+$ and Calculated for $\mathrm{C}_{16} \mathrm{H}_{14} \mathrm{ClN}_{3}: 283.76$.

\section{RESULTS AND DISCUSSION}

A model reaction of 2,6-dichloro-quinoxaline with (6-chloropyridin-3-yl)methanol using $\mathrm{K}_{2} \mathrm{CO}_{3}$ was employed under the conventional stirring method (Scheme-2.) at $50-55^{\circ} \mathrm{C}$ in DMF medium. The expected product was yield in very low and the reaction not completed, even extending reaction time up to $24 \mathrm{hrs}$. This experiment result encouraged us to explore new ideas for better reaction conditions to get good yields. The reaction temperature increased to $70-75^{\circ} \mathrm{C}$ to obtained better yield $(80 \%)$ in $12 \mathrm{hrs}$. While 
RASĀYAN J. Chem.

Vol. 13 | No. 2 |1037 - 1041| April - June | 2020

doing further optimization study it was observed that introducing of phase transfer catalyst such as Tri Ethyl Benzyl Ammonium Chloride (TEBAC) makes the completion of the reaction within 4 hrs with increased yields at $70-75^{\circ} \mathrm{C}$ (Table-1). The scope of this protocol was extended to a variety of novel substituted alcohols and thiols (Table-1, entries 1-5). After making derivatives with phenols and thiols, we thought to introduce nitrogen atom in the second position of Quinoxaline. To explore this chemistry and the activity of the amino derivative, we tried a reaction with 2,3-dimethylaniline, DMF as solvent without any base obtained afford corresponding substituted derivative (Table-1, entry 6). The newly synthesized six compounds were successfully characterized by spectral data such as IR, ${ }^{1} \mathrm{HNMR}$, ${ }^{13} \mathrm{CNMR}$ and mass spectrometry.

Table-1: Optimization Reaction Condition for Preparation of 3a

\begin{tabular}{c|c|c|c}
\hline \multirow{2}{*}{ Entry } & Reaction Condition & $\begin{array}{c}\text { Raction } \\
\text { Time }(\mathrm{hr})\end{array}$ & ${\text { Yield }(\%)^{\mathrm{a}}}$ \\
\hline 1 & $\mathrm{RT}$ & 24 & 28 \\
\hline 2 & $50-55^{\circ} \mathrm{C}$ & 24 & 52 \\
\hline 3 & $60-65^{\circ} \mathrm{C}$ & 18 & 65 \\
\hline 4 & $70-75^{\circ} \mathrm{C}$ & 12 & 76 \\
\hline 5 & $80-85^{\circ} \mathrm{C}$ & 12 & 80 \\
\hline 6 & $100^{\circ} \mathrm{C}$ & 12 & 78 \\
\hline 7 & $\mathrm{TEBAC}, 60-65^{\circ} \mathrm{C}$ & 8 & 69 \\
\hline 8 & $\mathrm{TEBAC}, 70-75^{\circ} \mathrm{C}$ & 4 & 84 \\
\hline 9 & $\mathrm{TEBAC}, 80-85^{\circ} \mathrm{C}$ & 4 & 82 \\
\hline
\end{tabular}

${ }^{a}$ isolated yield

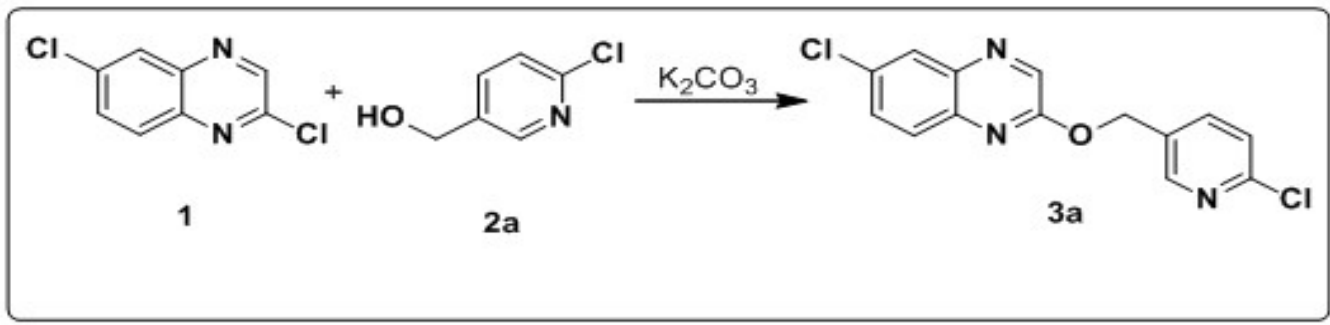

Biological Activity

Scheme-2: Optimization Reaction Condition for Preparation of 3a

In vitro antimicrobial activity of the compounds (3a-f) were evaluated using Agar well-diffusion method against four different organisms two Gram-positive bacteria and two Gram-negative bacteria such as $S$. aureus (ATCC-6538), B. subtilis (ATCC-6633), E. coli (ATCC-11229) and P. aeruginosa (ATCC29213). Chloramphenicol was used as a standard drug. The antibacterial study results in a zone of inhibition mentioned in Table-2. The antibacterial results show that the replacement of 6-chloropyridine ring by 2-chlorothiazol group and 2,3-dimethylaniline, activity has been enhanced against all tested bacteria. The compounds 3c, 3d and $\mathbf{3 f}$ showed better activity compared to the standard drug against tested all organisms (Table-2). The compounds $\mathbf{3 c}$ and $\mathbf{3 d}$ showed maximum antibacterial activities that may responsible for the 2-chlorothiazide group.

Table-2: Antibacterial Activity of 2-Substituted 6-Chloro-Quinoxaline Derivatives (3a-f)

\begin{tabular}{c|c|c|c|c}
\hline Compound & \multicolumn{2}{|c|}{ Gram-positive Strains } & \multicolumn{2}{c}{ Gram-negative Strains } \\
\hline & Bacillus subtilis & $\begin{array}{c}\text { Staphylococcus } \\
\text { aureus }\end{array}$ & $\begin{array}{c}\text { Pseudomonas } \\
\text { aeruginosa }\end{array}$ & Escherichia coli \\
\hline 3a & 12.0 & 14.0 & 13.0 & 11.0 \\
\hline 3b & 11.0 & 12.0 & 14.0 & 13.0 \\
\hline 3c & 22.0 & 23.0 & 24.5 & 21.0 \\
\hline 3d & 24.0 & 23.0 & 23.0 & 10.0 \\
\hline 3e & 10.0 & 11.5 & 12.0 & 22.0 \\
\hline 3f & 23.0 & 24.0 & 25.0 & 20.0 \\
\hline Chloramphenicol & 25.0 & 24.0 & 22.0 & \\
\hline
\end{tabular}


RASĀYAN J. Chem.

Vol. 13 | No. 2 |1037 - 1041| April - June | 2020

\section{CONCLUSION}

We have successfully synthesized novel 2-Substituted-6-chloroquinoxalines by using a phase transfer catalyst. The present method is economically feasible because the products can be obtained through a facile, easy and efficient. The compounds showed $\mathbf{3 c}$ and $\mathbf{3 d}$ showed promising antibacterial activity and the remained all compounds showed moderate activity.

\section{ACKNOWLEDGMENT}

The authors are thankful to the Chairman, Vignan University, Guntur, India and the Managing Director, NACL Industries 1td, Hyderabad, India, for the financial support and providing laboratory facilities to carry out the research work.

\section{REFERENCES}

1. R.V. Ghadage and P.J. Shirote, Journal of Chemical and Pharmaceutical Research, 3(5), 260(2011)

2. Mao Zhang, Zhi-Cheng Dai, Shao-Song Qian, Jun-Yan Liu, Yu Xiao, Ai-Min Lu, Hai-Liang Zhu, Jian-Xin Wang and Yong-Hao Ye, Journal of Agricultural and Food Chemistry, 62(40), 9637(2014), DOI: $10.1021 / \mathrm{j} 5504359 \mathrm{p}$

3. A.K. Parhi, Y.Z. Zhang, K.W. Saionz, P. Pradhan, M. Kaul, K. Trivedi, D.S. Pilch and E.J. LaVoie., Bioorganic \& Medicinal Chemistry Letters, 23, 4968(2013), DOI:10.1016/j.bmcl.2013.06.048

4. A.M.S.E. Newahie, Y.M. Nissan, N.S.M. Ismail., D.A. Abou, El. Ella, S.M. Khojah, and K.A.M. Abouzid, Molecules, vol. 24, 1175(2019), DOI:10.3390/molecules24061175

5. M. Santivanez-Veliz, S. Perez-Silanes, E. Torres and E. Moreno-Viguri, Bioorganic \& Medicinal Chemistry Letters, 26, 2188(2016), DOI:10.1016/j.bmcl.2016.03.066

6. J.L. Sessler, H. Maeda, T. Mizuno, V.M. Lynch and F. Hiroyuki, Journal of the American Chemical Society, 124, 13474(2002), DOI:10.1021/ja0273750

7. S. Chakraborty, S. Goswami, C. K. Quah and B. Pakhira, Royal Society Open Science, 5(6), 180149(2018), DOI: 10.1098/rsos.180149

8. P. Ramalingam, K. Rajendran, K. Sunil Kumar and Y. Padmanabha Reddy, International Journal of Medicinal Chemistry, 6471352, 8(2016), DOI:10.1155/2016/6471352

[RJC-5693/2019] 